\title{
A FARADAY ROTATION SURVEY IN THE FIRST QUADRANT OF THE GALAXY
}

\author{
ANDREW CLEGG and JAMES CORDES \\ Department of Astronomy \\ Cornell University \\ Ithaca, New York 14853 \\ $U S A$
}

\begin{abstract}
We have measured the degree of Faraday rotation towards 73 extragalactic sources at low Galactic latitudes, predominantly within the first quadrant of longitude. The Faraday rotation towards extragalactic sources follows the trends of that towards pulsars, a fact which vindicates an initial assumption that the rotation arises mostly from within the Galaxy. When combined with the pulsar data, our data imply the existence of magnetic field reversals along several lines of sight. Extragalactic sources with $l>60^{\circ}$ have rotation measures that are systematically larger than those for pulsars in the same region, which suggests that the structure responsible for the excess rotation must lie more distant than $\sim 7 \mathrm{kpc}$.
\end{abstract}

\section{Introduction}

The rotation measure is proportional to the integrated electron density and magnetic field component along the line of sight:

$$
R M=C \int_{\text {los }} n_{e} B_{\|} \mathrm{d} l
$$

The integrand represents the product of the electron density, $n_{e}$, the parallel component of the magnetic field, $B_{\|}$, and a differential pathlength $\mathrm{d} l$ along the line of sight. For $n_{e}$ in units of $\mathrm{cm}^{-3}, B_{\|}$in $\mu$ gauss, and $\mathrm{d} l$ in $\mathrm{pc}, C=0.81$.

\section{Observations and Data Analysis}

Polarization position angles, $\theta$, were measured at six frequencies throughout the $20 \mathrm{~cm}$ band using the Very Large Array radio telescope. The angles were measured in the range $\pm \pi / 2$ and were later shifted in increments of $\pi$ by up to $\pm 2 \pi$ to produce a minimum $\chi^{2}$ fit in the position angle vs. $\lambda^{2}$ plane. The resultant line slope yielded the rotation measure, $R M$, defined by

$$
R M \equiv \frac{\mathrm{d} \theta}{\mathrm{d}\left(\lambda^{2}\right)}
$$




\section{Analysis}

Most of our measurements lie in the range $30^{\circ}<l<100^{\circ}$. In this region a systematic component to the rotation measure is apparent, with $\mathrm{RM}$ running from large positive values at $l \sim 40^{\circ}$, to large negative values at $l \sim 90^{\circ}$ (see figure 1). This behavior is probably caused by the transition of the line of sight through the Galaxy passing from tangential to the Sagittarius spiral arm, through a void between spiral arms near $l \sim 55^{\circ}$, to a direction along the Perseus arm at $l \sim 90^{\circ}$.

The rotation measures towards our sample of extragalactic sources can be compared with those measured towards pulsars. In general, the pulsar data do not show the large negative values in the range $60^{\circ}<l<90^{\circ}$. Apparently, this negative component arises at some distance greater than that at which the observed pulsars lie. The pulsar sample includes distances out to approximately $7 \mathrm{kpc}$, thus we conclude that a significant component of the rotation occurs at a greater distance.

Several of the extragalactic sources have more than one component, making it possible to discern rotation measure differences on scales as small as $\sim 6$ arc sec. Some multi-component sources show nearly constant rotation measures across all components, while others show differences as great as $\sim 300 \mathrm{rad} \mathrm{m} \mathrm{m}^{-2}$ over a few arc sec. Presumably such differences are due to the effects of discrete structures such as supernova remnants, HII regions, and dense molecular clouds.

\section{Summary}

A preliminary analysis of the data yields the following conclusions:

- The rotation measures are orderly on large angular scales $\left(\sim 45^{\circ}\right)$ indicative of the spiral arm structure of the Galaxy.

- Small-scale ( $\sim$ few arcsec) variations can be large. Some rotation measures are presumably dominated by discrete structures in the ISM, including supernova remnants, HII regions, and dense molecular clouds.

- The rotation measures of extragalactic sources with $0^{\circ}<l<60^{\circ}$ agree favorably in amplitude and sign with those measured for pulsars in the same region.

- Extragalactic sources with $l>60^{\circ}$ have rotation measures that are systematically larger in amplitude than those for pulsars in the same region. The structure responsible for producing the excess rotation must lie farther than the distance to the observed pulsars (farther than roughly $7 \mathrm{kpc}$ ).

- Along several lines of sight that are coincident to within $5^{\circ}$, the rotation measures of extragalactic sources appear to be consistent with extrapolated values of those observed towards pulsars of increasing distance. For $l \sim 55^{\circ}$ and distance $\sim 5$ kpc (approximately the tangent point to the Sagittarius arm), there appear to be significant reversals of rotation measures in the pulsar data.

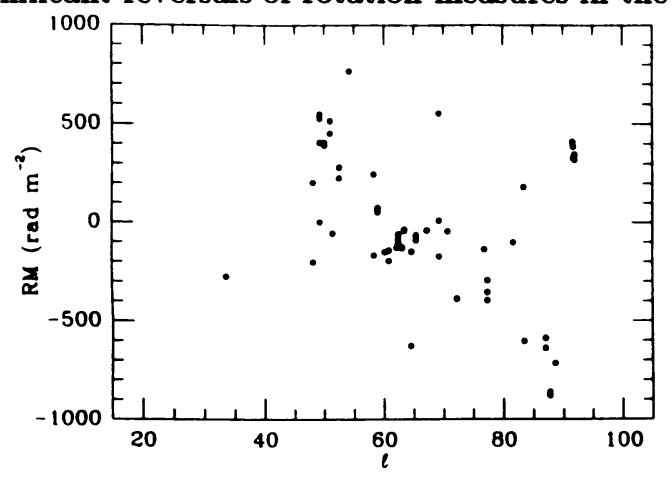

Figure 1. Rotation measures as a function of Galactic longitude. Many of the data points represent multi component sources whose separate components have similar rotation measures. A total of 73 lines of sight were sampled. 